\title{
Compte rendu de l'activité de la Commission pour l'Étude des Débits ")
}

\author{
a) Extension des travaux à certaines questions telles que l'étude des basses eaux \\ b) Réunion du 25 octobre 1963 sur les débits d'étiage
}

\section{Activity Report of the "Discharge Studies Commission" "**)}

a) Extension of the Commission's work to include a study of low water conditions and other questions

b) Meeting of the 25 th October on low-water discharge

PAR Ph. DEYMTÉ,

INSPECTEUR GÉNÉlLAL DES PONTS ET CHAUSSÉES

PRÉSIDENT DE LA COMMISSION POUR L'ÉTUDE DES DÉBITS A LA S.H.F.

\begin{abstract}
Il est difficile de séparer l'étude des débits de crues d'une rivière de l'étude des autres débits caractéristiques de cette rivière. En particulier les courbes de tarissement interviennent dans l'établissement de l'hydrogramme unitaire. D'autre part, du fait de la progression rapide des besoins en eau, l'étude des débits d'étiage prend actuellement une importance particulière. Pour ces raisons, la Commission pour l'élude des débits de crues a cru devoir étendre le domaine de ses investigations, elle a tenu, le 25 octobre, une réunion qui était consacrée à l'étude des débits d'étiage.

Le programme de cette réunion compronait deux parties:

$1^{\circ}$ Généralités sur l'occurrence, la mesure et le traitement statistique des débits d'étiage, avec la collaboration de MM. Tschirhardt (Météorologie), Goubet (1'e Circonscription Electrique) et Bernier (E.d.F.).

$2^{\circ}$ Etat des connaissances des débits d'étiage sur les principaux bassins fluviaux français (Seine en 1961 et 1962 , par M. Goubet de la $1^{\text {re }}$ Circonscription Electrique, Moselle en 1959 et 1962, Meuse de $1953 \dot{\alpha} 1962$, par MM. Lebreton et Diconne de la $2^{\circ}$ Circonscription Electrique) ou dans certaines zones critiques de ces bassins (Rhin $\dot{a}$ Bàle, par M. Jacob, d'E.d.F., Rhône d̀ Châteaufort, La Mulatière et Valence, par $M$. Mauchamp de la C.N.R., Loire en aval du Bec d'Allier, par MM. Pellecuer et Barrière, de la $4^{\mathrm{e}}$ Circonscription Electrique).
\end{abstract}

(*) Anciennement : Commission pour l'Etude des Débits de Crues.
It is difficult to study the flood discharge of a river separately from its other characteristic flows. In particular, recession curves have to be considered in drawing ap the unit hydrograph. Furthermore, in view of the rate at which water requirements are now increasing, the study of low-water discharge has assumed a special importance.

The Commission considered it advisable, therefore, to extend the field of its investigations to also include low-water discharge, and a meeting was helds on this subject on the 25 th $O \mathrm{Cc}_{\text {- }}$ tober, with a two-part programme covering the following:-

1. General remarks on the occurrence, measurement and statistical analysis of low-water flows, with contributions by Mr. Tschirhardt (Météorologie Nationale), Mr. Goubet (1 ${ }^{\mathrm{re}}$. Circonscription Electrique) and Mr. Bernier (Electricité de France);

2. A review of known low-water flow data for the main French river basins (The Seine in 1961 \& 1962 by Mr. Goubet of the "1 $1^{\text {re }}$ Circonscription Electrique", the Moselle in 1959 \& 1962 and the Meuse from 1953 to 1962 , by Mr. Lebreton and Mr. Diconne of the " 2 "Circonscription Electrique", and certain critical zones in these basins, i.e. the Rhine at Basle, by Mr. Jacob of E.d.F., the Rhone at Chateanfort, La Mulatierre and Valence, by Mr. Matuchamp of the "Compagnie Nationale du Rhone", and the Loire downstream from its confluence with the Allier, by Mr. Pellecuer and Mr. Barriere of the "4. Circonscription Electrique"). 
Depuis 1961, la Commission pour l'Etude des Débits a organisé trois sessions:

- L'une, consacrée aux crues d'octobre 1960 dans le centre de la France, a eu lieu le 15 mai 1961 et ses conclusions, présentées au Comité technique de la S.H.F., ont été adoptées le 16 juin 1961.

- La seconde, tenue le 4 mai 1962, avait pour objet de préciser certains problèmes évoqués à l'occasion des communications présentées en 1961, notamment en ce qui concerne l'estimation des débits maximaux de crues (problème de l'extrapolation des courbes de tarage expérimentales et évaluation a posteriori des débits à partir d'observations dans des sections singulières de rivières) et des difficultés d'étude statistique de la distribution des débits de crues.

- La troisième réunion, toute récente puisqu'elle a eu lieu le 25 octobre 1963 , a eu pour thème l'étude des basses eaux - autrement dit des débits d'étiage. Il est en effet apparu, à la suite de la sécheresse de l'été et de l'automne 1962, l'intérêt de certaines études ayant pour objet la mise au point des techniques d'étude des bas débits et le rassemblement de l'information les concernant. Comme la Commission d'étude des débits yroupe pratiquement l'ensemble des hydrologues que la question intéresse, il était donc superflu de créer à cette occasion une nouvelle commission et l'étude des débits est passée d'un extrême à l'autre...

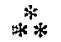

Ce sont les travaux et conclusions des deux dernières réunions de 1962 et 1963 qui feront principalement l'objet de ce compte rendu d'activité.

$$
* * *
$$

A la suite de la réunion de 1961 , les principales directions de recherches retenues pour les travaux ultérieurs de la Commission étaient les suivantes :

1. Dans le domaine de la prévision météorologique des crues :

- La recherche et l'analyse de situations météorologiques types génératrices de crues sur les différents bassins fluviaux;

- l'examen approfondi des possibilités d'utilisation des radars météorologiques pour la localisation des maximums pluviométriques.

\section{Dans le domaine de l'annonce des crues :}

- les dispositifs de télémesure pluviométriques et limnimétriques et de transmission des observations hydro-météorologiques;
- la comparaison des méthodes de mesure directe et d'estimation indirecte des débits de crues en vue du choix et de l'aménagement des stations de jaugeage de crues;

-. le calcul des hydrogrammes de crues à partir de la pluie;

- l'étude de la propagation des ondes de crues.

\section{Dans le domaine de la protection contre les crues :}

-.- Ia définition des «zones critiques» à protéger et plus précisément des critères à prendre en considération pour cette définition;

- l'étude probabiliste des phénomènes exceptionnels.

Cette énumération, incomplète puisqu'elle ne concerne que les crues d'origine pluviale et n'aborde pas la question de la fusion nivale, délimite un cadre de travail extrêmement vaste tant par la nature des problèmes soulevés que par l'éventail des compétences auxquelles il faut faire appel pour les traiter, depuis celle du météorologiste jusqu'à celle de l'économiste.

II est entendu que le but des travaux de cette Commission est moins de «fixer» une doctrine ou des méthodes d'étude encore en évolution que de faire le point de l'avancement des techniques.

Ainsi il est bien évident que la question du calcul des hydrogrammes à partir de la pluie devra être reprise et précisée au fur et à mesure des progrès accomplis dans ce domaine.

Une certaine continuité doit donc être assurée dans les travaux de la Commission, et ceux qui ont fait l'objet de la réunion de 1962 s'inscrivent logiquement dans le cadre défini plus haut.

Cinq rapports ont été présentés à cette réunion :

- La communication de M. Plancher, Ingénieur à la Division Technique Générale (E.D.F.), sur les pluies exceptionnelles et la crue de la Cère des 12 et 13 janvier 1962 , relève du domaine de la prévision hydrométéorologique. Les études monographiques de crues importantes telles que celle-ci, qui a atteint $600 \mathrm{l} / \mathrm{s}: \mathrm{km}^{2}$ à Saint-Etienne-Cantalès pour un bassin versant de $691 \mathrm{~km}^{2}$ ), sont maintenant l'occasion de recherches et de comparaison des situations météorologiques types génératrices de crues sur une zone déterminée et contribuent à améliorer la prévision de ces phénomènes;

- Les crues «exceptionnelles» qui ont affecté le Massif Central en octobre 1960 ont reposé le problème de la probabilité à attribuer à ces événements et des difficultés du choix d'un support d'extrapolation pour la loi de probabilité des débits maximaux ont été clairement mises en évidence à cette occasion. Deux 
communications ont apporté à ces questions de nouveaux éléments de solution :

$1^{\circ}$ M. Guillo', Chef du Service hydrométéorologique de la Division Technique Générale (E.D.F.), en présentant ses réflexions sur la probabilité du débit maximal annuel et ses relations avec la loi de distribution des débits journaliers, a montré que la loi du plus fort débit journalier de l'année pouvait être dotée d'une asymptote déduite de la courbe des débits classés dans un système de coordonnées particulier. En écartant certaines objections de principe (non-indépendance des débits journaliers), on dispose ainsi d'un support d'extrapolation qui permet de surmonter les difficultés du choix entre divers types de lois de valeurs extrêmes;

$2^{\circ}$ Avec la communication de MM. Bernier et Véron, Ingénieurs à la Division Hydrologie du C.R.E.C. (E.D.F.), ce sont les problèmes d'estimation des quantiles d'une loi de probabilité qui sont examinés à propos de l'estimation d'un débit de crue correspondant à une probabilité donnée. L'essentiel de ce rapport sera d'ailleurs présenté au cours de la présente session du Comité technique.

- Les communications de M. André, Chef du Service des Etudes et Mesures Hydrométriques de la Division Technique Générale (E.D.F.) et de M. Estienne, Ingénieur des Ponts et Chaussées à la $5^{\mathrm{e}}$ Circonscription Electrique (Toulouse), concernent les travaux d'un groupe de travail qui a été constitué pour faire le point des méthodes d'estimations - autres que le jaugeage direct - des débits maximaux de crues: problème de l'extrapolation des courbes de tarage expérimentales et des estimations a posteriori à partir de relevés d'observations hydrométriques dans des sections singulières de cours d'eau, telles que seuils ou étranglements.

La constitution de ce groupe de travail appelle quelques commentaires. Le bon fonctionnement d'une commission d'étude aussi nombreuse que la nôtre pose en effet un problème de méthode de travail. Nous avons donc réuni, pour l'étude d'unè question bien délimitée : savoir, les méthodes d'estimation des débits de crues, un groupe de travail restreint comprenant une dizaine de personnes spécialistes d'hydrométrie ou intéressées directement par le problème. La première réunion de ce groupe, le 19 février 1962, a permis de définir les véritables contours du problème étudié. Les échanges d'informations qui ont eu lieu sur les méthodes d'estimation de débits de crues utilisées dans les divers services intéressés ont abouti à l'organisation d'une enquête auprès des organismes exploitants de stations de jaugeage, en vue d'étudier, sur des valeurs expérimentales suffisantes de coefficients de rugosité, dans quelle mesure la formule de Strickler-Manning peut être utilisée pour le recoupement de valeurs de débits maximaux de crues estimées par d'autres méthodes et, par suite, être utilisée pour l'extrapolation des courbes de tarages.

Les résultats de l'enquête seront discutés au sein du Groupe de travail avant d'être soumis à la Commission. Force est toutefois de constater que le questionnaire présenté n'a guère reçu de réponses à cette heure.

Le choix de ce problème, parmi ceux qui avaient été retenus à la suite des réunions de 1961, répond à une préoccupation d'ordre méthodologique : la question de la précision d'évaluation des débits maximaux de crues doit logiquement précéder les études de distribution géographique, dans le temps et par importance, de ces phénomènes. II devrait résulter des travaux du Groupe de travail la rédaction d'un document faisant le point des différentes techniques et de leurs domaines d'utilisation, document qui résumerait l'expérience acquise à ce jour dans les divers services hydrométriques en matière d'estimation des débits de crues et d'aménagement des sections de mesures. Les rapports présentés par M. ANDRE et M. Estienne passent en revue les diverses méthodes mises au point pour l'extrapolation des courbes de tarages expérimentales dans les Services hydrométriques, et constituent ainsi la base de cette documentation.

$$
* *
$$

Le nouveau champ d'activité ouvert à la Commission d'étude des débits en 1963 répond aux impératifs suivants :

- La question des débits de basses eaux des cours d'eau prend une importance croissante, principalement dans les zones a forte concentration industrielle, où des prélèvements substantiels aggravent la pollution et où l'on utilise l'eau courante comme source froide d'installations thermiques;

- Il est donc nécessaire que les ingénieurs aient a leur disposition des informations générales et claires sur le crédit à accorder aux données d'observation concernant les bas débits, sur les difficultés rencontrées pour leurs mesures et sur les méthodes d'étude des étiages:

-... Enfin, il s'avìre utile de rassembler sous une forme assez directement utilisable une première documentation relative aux bas débits des principaux fleuves français en certains points critiques, du point de vue besoins en eau, de leurs cours. Cette documentation constituée à partir des données disponibles sera ensuite complétée et mise à jour au fur 
et à mesure des progrès d'observation et de mesure de basses eaux.

Pour réaliser ces objectifs, et en particulier pour dégager une méthodologie d'étude, nous avons procédé d'une façon analogue à celle qui a fait ses preuves pour l'étude des crues en demandant à des «professionnels»:

d'une part, d'exposer leur point de vue de spécialistes sur la météorologie des sécheresses, la mesure des débits d'étiage dans les rivières de plaine canalisées, et la probabilité des sésécheresses et des bas débits;

d'autre part, de présenter sur quelques exemples (Seine, Meuse, Moselle, Rhin, Rhône, Loire) un état de la question des étiages d'après l'information expérimentale existante.

D'où les deux parties de la réunion du 25 octobre 1963 :

1. Généralités sur l'occurrence, la mesure et le traitement statistique des débits d'étiage;

2. Etat des connaissances des débits d'étiage sur les principaux bassins fluviaux français ou certaines zones critiques de ces bassins.

Au cours de la $1^{\text {re }}$ partie de cette réunion, trois rapports ont été présentés :

- Une introduction à l'étude des sécheresses par M. Tschirhart, Ingénieur de la Météorologie, détaché à E.D.F., qui, analysant les causes des périodes sans pluie, en distingue des causes physiques (absence de vapeur d'eau atmosphérique du fait du froid ou de la continentalité), une cause dynamique (anticyclone qui constitue une zone de subsidence, e'est-à-dire un effondrement continu vertical des masses d'air se traduisant par une augmentation des températures des basses couches et par la formation d'inversions de température) et une cause aérologique (présence d'air chaud d'origine advective dans les couches moyennes). La conjugaison de ces divers types de causes explique la distribution géographique et temporelle des sécheresses. Remarquant qu'en définitive, toute sécheresse est d'origine advective, M. Tschirhart en tire des conclusions sur la possibilité de définir des critères de prévision de ces phénomènes et sur une méthode de classification des étiages des cours d'eau français par analyse des situations météorologiques qui en sont responsables.

- Les difficultés rencontrées pour la mesure des débits d'étiage sur les cours d'eau canalisés ont été ensuite exposées par M. Gouber, Ingénieur des Ponts et Chaussées à la $1^{\mathrm{re}}$ Circonscription Electrique (Paris), dont le Service exploite les stations de jaugeage du bassin de la Seine et des cours d'eau du nord. Sur de telles rivières, la détermination des débits est souvent impossible à partir des cotes du plan d'eau, dont les variations sont artificiellement effacées. Deux procédés de mesure sont présentés :

- l'un à partir de la mesure de la pente superficielle (ce qui impose des stations à 2 limnigraphes suffisamment éloignés - plusieurs kilomètres - dont l'exploitation se révèle très délicate : 8 stations de ce type sont actuellement en service à la $1^{\text {re }}$ C.E.);

- l'autre procédé consiste à déduire le débit de la vitesse en un ou quelques points de la section de jaugeage, dont on s'est assuré qu'ils sont «représentatifs » de la vitesse moyenne dans la section à un facteur constant près (cette méthode en est à ses débuts de réalisation pratique au moyen de jaugeages sommaires qui devraient permettre d'améliorer sensiblement la précision de mesure des débits à partir de la pente superficielle).

Toutefois le problème de l'étalonnage des stations de mesure pour les bas débits à faibles vitesses d'écoulement (quelques $\mathrm{cm} / \mathrm{s}$ ) reste primordial et $M$. Goubet fait part d'un certain nombre d'améliorations apportées au matériel de jaugeage pour l'adapter aux conditions de ces mesures (en particulier un moulinet à faible inertie entraîné par un courant de $2 \mathrm{~cm} / \mathrm{s}$ ).

- Le $3^{e}$ rapport, présenté par M. Bernier, Ingénieur à la Division hydrologie du C.R.E.C. (E.D.F.), sur les probabilités d'occurrence des sécheresses et des étiages, précise la nature aléatoire des indicateurs de sécheresse qui se présentent sous forme de séries chronologiques d'observations de durée finie. Les recherches de périodicité dans ces séries sont dénuées de fondément (faussées à la base par l'effet Slutsky) de même que celle des tendances à long terme en raison de la limitation en durée des séries que l'on analyse. Par contre, le perfectionnement des modèles probabilistes propres à décrire les faits observés peut être poussé assez loin, la seule limitation à la souplesse de ces modèles étant l'information réelle contenue dans les échantillons dont on dispose pour estimer les paramètres d'ajustement. M. BERNIER indique à ce propos un certain nombre de modèles, théoriquement plus réalistes que des schémas plus simples, pour décrire l'hydraulicité des années successives par exemple, mais qui ne peuvent être retenus par manque d'information suffisante. Comment représenter la dépendance en chaîne d'années successives (successions de sécheresses)? Cette question est d'un grand intérêt pour les prévisions et devrait être approfondie. En ce qui concerne plus spécifiquement les débits maximaux de cours d'eau et leur distribution, M. Bernier propose une méthode de calcul de la loi de probabilité des débits en fonction des lois de probabilités de facteurs météorologiques 
qui les conditionnent, et qui prend en compte la dépendance en chaîne des phénomènes.

La $2^{\circ}$ partie de la session a été consacrée à une présentation de résultats concernant les étages sur les principaux bassins fluviaux francais qui ont été affectés par les sécheresses des dernières années :

- La Seine est actuellement équipée d'un réseau suffisant de stations de jaugeage pour permettre l'analyse des étiages par sections de son cours et sur ses affluents, mais leur étalonnage médiocre pour les bas débits et leur faible ancienneté constituent encore des obstacles majeurs aux évaluations des probabilités de basses eaux dans la région parisienne. M. GoubeT a présenté un tableau détaillé des étiages sur le bassin de la Seine en 1961 et 1962, mettant en évidence que les débits moyens minimaux de 10 et 30 jours consécutifs peuvent descendre en dessous de $1 \mathrm{l} / \mathrm{s}$. $\mathrm{km}^{2}$ et que la période de basses eaux peut se prolonger très tard en automne. Une intervention de M. le Professeur Chartier a montré l'importance des observations suivies d'évolution des nappes profondes pour apprécier la tendance de la succession des étiages annuels.

- La connaissance des étiages de la Meuse à Chooz présente une importance particulière en raison de l'implantation en ce point d'une centrale nucléaire : on a la chance de disposer là d'une station de jaugeage stable et bien tarée pour les basses eaux. M. LEBREron, Ingénieur des Ponts et Chaussées et M. Diconne, Ingénieur T.P.E. à la $2^{n}$ Circonscription Electrique (Dijon), ont présenté une étude des basses eaux de la Meuse à la limite du territoire français, portant sur 10 années d'observations. I a méthode d'analyse séquentielle utilisée est particulièrement bien adaptée à l'étude des bas débits, qui peuvent être soumis à d'importantes fluctuations à l'échelle journalière et dont l'époque d'occurrence n'est pas indifférente : on recherche, pour chaque année, les valeurs minimales des moyennes mobiles des débits de 10, 30, 90 jours consécutifs, leurs périodes d'occurrence, et les valeurs de ces débits moyens atteintes ou dépassées 1 année sur 2, 4 années sur 5 et 9 années sur 10. Cette analyse fait apparaitre pour la Meuse des débits d'étiage minimaux de 10 jours de l'ordre de $2 \mathrm{l} / \mathrm{s} . \mathrm{km}^{2}$, avec le mois d'octobre comme période de basses eaux la plus fréquente.

- Le problème des basses eaux de la Moselle intéresse à la fois les zones industrielles traversées et la navigation : MM. LeBRETON et DiconNE: ont analysé les sécheresses de 1959 et 1962 qui ont affecté la Moselle, d'après la méthode mise en cuvre pour l'étude de la Meuse. Il apparaît notamment que la sévérité de l'étiage 1962 a été plus forte sur le bassin supérieur, tandis qu'en 1959, le cours moyen de la Moselle a connu les plus bas débits : ce fait doit être mis en relation avec la sécheresse exceptionnelle qui a affecté l'Allemagne cette année-là.

- Avec l'étude des basses eaux du Rhin à Bâle présentée par M. JACOB, Ingénieur à la Division Technique Générale (E.D.F.), on entre de plain-pied dans le traitement statistique de très longues séries d'observations puisque les débits du Rhin y sont connus depuis 1808. M. JACOB passe en revue les problèmes que pose l'analyse de ces séries (homogénéité des observations perturbée par l'effet des réservoirs et ouvrages de régularisation des lacs à l'amont, choix de la périodicité annuelle de base pour l'étude des étiages) et étudie les distributions de diverses caractéristiques des débits d'étiage (débit journalier minimal annuel, débit maximal des $n$ jours - consécutifs et non consécutifs - de plus bas débit, nombre de jours où le débit est resté inférieur à une valeur donnée). Les incidences des basses eaux sur la navigation rhénane à l'aval de Bâle rendent souhaitable la réalisation d'études communes par les Services de Navigation et les organismes de gestion des lacs suisses : la période d'hiver 1962-1963 a, en effet, été critique sur le Rhin, malgré les restitutions des réservoirs amont, battant les records de durée des basses eaux des hivers 1908-1909 et 19201921. L'occurrence des étiages du Rhin, qui nécessitent la conjugaison des eflets d'un été très sec avec une sécheresse d'automne prolongée en hiver, permet d'en envisager une prévision, basće sur l'inertie du sytème hydrographique, au moyen d'un indice représentatif des réserves liquides du bassin au début de la période normale des basses eaux.

- Sur le Rhône, où l'automne 1962 a connu des basses eaux d'une sévérité exceptionnelle à l'aval de Lyon, M. Mauchamp, Ingénieur à la Compagnie Nationale du Rhône, a analysé les éliages de l'amont vers l'aval en 4 stations choisies pour représenter les variations de régime du Rhône : Sault-Brenaz à l'amont du confluent de l'Ain (régime nivo-glaciaire), Lyon-La Mulatièro (qui intègre les apports pluviaux de l'Ain et de la Saône), Valence (influence des réservoirs de l'Isc̀re), Beaucaire (cours d'eau cévenols : Ardèche et Gard, et méditerranéens : aménagements de la Durance). Ces stations permettent de défnir les débits minimaux hebdomadaires qui se sont produits en décembre 1962 à Sault-Brena\% avec $12,85 \mathrm{l} / \mathrm{s} . \mathrm{km}^{2}$ et en octobre à l'aval : $4,64 \mathrm{l} / \mathrm{s} . \mathrm{km}^{2}$ à Lyon (étiage de la Saône: $1,64 \mathrm{l} / \mathrm{s} . \mathrm{km}^{2}$ ) - $6,32 \mathrm{l} / \mathrm{s} . \mathrm{km}^{2} \dot{a}$ Valence (influence des réservoirs de l'Isère) et $5,28 \mathrm{l} / \mathrm{s} . \mathrm{km}^{2}$ à Beaucaire (prélèvements pour irrigation et réservoirs de la Durance). La comparaison de ces chiffres avec le passé est rendue délicate par les nombreux aménagements réalisés sur les hauts bassins (réservoirs) et à l'aval de Lyon (navigation). Les basses eaux d'automne 1962 doivent 
être rapprochées de celles de 1921 avec les particularités suivantes : 1921 est plus sévère à l'amont de Lyon; à l'aval du confluent Saône entre Lyon et le confluent de l'Isère, le record est détenu par 1962; à Valence et à Beaucaire, les débits observés les plus faibles sont ceux de 1921 mais, après correction du jeu de réservoirs, c'est 1962 qui l'emporte. Ce dernier point fait ressortir l'importance du jeu des réserves de l'Isère et de la Durance pour le soutien des étiages du bas Rhône, où 1962 constitue un record d'au moins 60 ans.

- C'est un exemple de valorisation d'une longue série d'observations non exploitée jusqu'ici que présentent $M$. Pellecuer, Ingénieur des Ponts et Chaussées et M. Barrière, Ingénieur T.P.E. à la $4^{*}$ Circonscription Electrique (Limoges) à propos des étiages de la Loire à l'aval du Bec d'Allier. Quatre stations de cette section du cours de la Loire disposent de plus de 100 années de lectures d'échelles, et des jaugeages de bonne qualité, effectués par intermittence en $1857-1874,1900-1910,1920$ et depuis 1929, permettent de situer les grands étiages, notamment ceux de1870, 1921 et 1949. MM. Pellecuer et Barrière ont ainsi pu reconstituer une série de 100 années de débits de la Loire à Blois, contrôlés par recoupement et corrélation avec les stations voisines. La sévérité des étiages étant caractérisée par la durée pendant laquelle le débit est resté inférieur à une valeur donnée et par les valeurs minimales des débits moyens de 20 et 30 jours successifs, l'année le plus déficitaire observée au cours d'un siècle (1862-1963) est 1949 (débit minimal $0,5 \mathrm{l} / \mathrm{s} . \mathrm{km}^{2}$ à Blois) suivie de 1870 et de 1921 . L'étiage décennal de 20 jours consécutifs est de l'ordre de $1,11 / \mathrm{s.km}^{2}$. Cette étude a été l'occasion d'intéressantes précisions sur le problème des pertes de la Loire: entre Gien et Orléans, on peut estimer ces pertes à 30 à $35 \%$ du débit à l'étiage et la récupération entre Orléans et Blois dépasse $20 \mathrm{~m}^{3} / \mathrm{s}$, imputables pour $50 \%$ environ au Loiret et pour $50 \%$ à des sources dans le lit ou à des apports souterrains de rive droite de la Loire.

Ces résumés ne peuvent donner une idée complète de la valeur de la documentation rassemblée par les différents rapporteurs. La variété des cas traités - tant du point de vue des régimes naturels des cours d'eau étudiés, des influences artificielles qu'ils subissent, que de la qualité des mesures et de la longueur des séries d'observation dont on dispose - fournit dès maintenant un échantillonnage assez complet des méthodes d'étude des basses eaux. Nous pensons qu'il serait utile de réunir dans une publication l'ensemble de ces communications, qui constituent une première documentation sur les étiages de cours d'eau français, dont l'intérêt, notamment pour les problèmes d'équipement et de navigation, est certain.

\section{***}

Ainsi, au domaine majeur d'activité de la Commission d'étude des débits — celui de l'étude des crues, pour lequel les directions de recherches définies en 1961 demeurent valables et feront l'objet de futurs travaux de la Commission - est venu s'adjoindre en 1963 l'étude des basses eaux, dont on peut prévoin d'importants développements notamment en ce qui concerne :

- la météorologie des sécheresses et la relation entre la pluviométrie et les étiages;

- l'étude des courbes de tarissement des cours d'eau en relation avec l'évolution des nappes souterraines;

- la recherche de modèles probabilistes représentatifs des phénomènes de basses eaux;

- les méthodes de mesure des débits aux faibles vitesses et d'exploitation de stations de mesure sur les cours d'eau canalisés;

- les monographies des étiages des principaux cours d'eau français.

$$
\text { ** }
$$

En terminant, je tiens à dire la grande part prise par M. JACQuet à l'activité de la Commission des Débits.

C'est M. JACquet qui cherche ef qui trouve les rapporteurs compétents pour la question sur laquelle chaque session de la Commission est centrée.

Je veux ici le remercier publiquement. 


\title{
D I S C US I I N
}

\author{
Président : M. RÉMÉNIÉnas
}

M. le Président remercie M. L’Inspecteur Général DEYMrk, qui, après nne belle série d'études sur les crues, vient d'ouvrix une voie très féconde avec l'nnalyse des débits d'étiage. Ces derniers premnent une importance économique grandissante dans bien des domaines, tels que l'irrigation, l'alimentation des circuits de condensation des centrales thermiques, etc.

M. Dexmié dit que cette importance devient de plus en plus grande, d'une part, à cause de la pollution et, d'autre part, à cause de l'utilisation de l'eau. Il est probable que les aménagements qui se feront dans les années à venir seront des aménagements te réservoirs, soit pour l'irrigation, soit pour le soutien du débit d'étiage.

M. le Président remarque que, de même que le gaz a été d'abord utilisé pour l'éclairage, application aujourd'hui disparue, il se peut qu'à longue échéance, la production de l'électricité ne soit plus qu'un sous-produit des aménagements hydrauliques, qui seront alors établis essentiellement pour la régulation des cours d'eau en vue de fournir en toute saison les débits nécessaires aux divers usages.

M. Deymé indique qu'il y a, actuellement, au Parlement, un projet de loi sur l'eau, qui a été voté par l'Assemblée Nationale et qui viendra probablement en discussion au Sénat au printemps. Si cette loi est définitivement votée, elle donnera des moyens juridiques nouveaux pour résoudre les problèmes techniques.

M. le Président souligne le difficile problème des stations de jaugeage pour la mesure des très faibles débits d'étiage, notamment pour les cours d'eau de plaine, souvent canalisés et ne présentant que des vitesses d'écoulement infimes; sur la Seine, par exemple, l'existence de barrages mobiles a rendu obligatoire l'installation de stations à deux échelles qui donnent des résultats acceptables pour les débits forts et moyens; mais ell étiage, la pente du plan d'eau est si faible que les mesures ainsi exécutées deviennent imprécises. M. Gouner tourne cette difficulté en reliant le débit du cours d'ean aux deux paramètres suivants :

- le nivenu du plan d'eau; et,
- la vitesse de l'écoulement en un point judicieusement choisi de la section.

En raison des faibles vitesses de l'écoulement, il utilise un moulinet spécial mesurant correctement des vitesses de $2 \mathrm{~cm} / \mathrm{s}$. Pour l'établissement de la famille des courbes de tarage dans ces cas difficiles, on peut songer à utiliser malgré leur complexité les tuyères électromagnétiques (1) qui sont sensibles à des vitesses de l'ordre du $\mathrm{mm} / \mathrm{s}$; quelques essais de se gense effectués par le Centre de Recherches et d'Essais de Chatou ont donné de bons résultats.

M. DExMIÉ dit que le problème est difficile, sans parler du matériel à mettre en auvre.

Sur des rivières canalisées, en général, quand le débit augmente dans une première période, la halutcur d'eau diminue, ćtant donné qu'on ouvre le barrage; et même, de plus en plus, il arrive que l'on fasse pivoler le niveau de la relenue autour d'un point situé en amont, de sorte que plus le débit augmente, plus le niveau baisse dans la zone anont du barrage. Il faut donc, ćvidemment, une relation hauteur-vitesse, mais en réalité, c'est la vitesse qui inlervient plus que la hatuteur. C'est la «double station» dont a parlé M. Govber.

M. DeYmé ajoute qu'en outre les mesures sont troublées par d'autres éléments, comme la navigotion, pendant le jour. M. Gouber a attiré l'attention sur ce point en disant que c'était pendant la nuit que l'on arrivait a l'état non perturbé, permettant de faire des mesures relativement exactes.

M. le Président remarque que l'on a pu espérer mesurer les très petits débits par les méthodes de dilution. Malheureusement le "bon mélange» du produit marqueur au sein de l'écoulement est difficile à rénliser lorsque les vitesses sont très faibles.

(1) G. Réméñénas el C. Hrnmaxt' : Mesure électronganétique des vitesses dans les Hiquides. La Houlle Blanche, B, 1954 . 